\title{
O PROCESSO DE ENFERMAGEM APLICADO AO PACIENTE COM FISSURA DE LÁBIO E/OU PALATO: REVISÃO INTEGRATIVA
}

\author{
Argemiro Alves da Silva Junior, Caroline Brandão Pires de Almeida \\ Universidade do Oeste Paulista - UNOESTE, Presidente Prudente, SP. e-mail: argemiroalvesir@hotmail.com
}

\section{RESUMO}

As fissuras labiopalatinas são consideradas as alterações de face mais frequentemente estudadas nas últimas décadas, em razão de sua grande incidência. $O$ objetivo desse estudo foi identificar a aplicação do processo de enfermagem aplicados a pacientes com fissura de lábio e/ou palato, descrita na literatura. Trata-se de revisão integrativa de abordagem qualitativa com foco na busca de resultados para a prática baseada em evidências. A partir da combinação dos descritores foram localizadas 38 produções. Após aplicação dos critérios estabelecidos a amostra final foi constituída de sete artigos. A implementação de um protocolo de cuidados ao portador de fissura, em diferentes contextos institucionais, e os resultados encontrados nessa revisão podem também inspirar a realização de futuros estudos que demonstrem o impacto que a assistência de enfermagem tem na reabilitação desses pacientes.

Palavras-chaves: fenda labial, fissura palatina, processo de enfermagem, diagnóstico de enfermagem, enfermagem.

\section{THE NURSING PROCESS APPLIED TO PATIENTS WITH CLEFT LIP AND/OR PALATE: AN INTEGRATIVE REVIEW}

\section{ABSTRACT}

Cleft lip and palate are considered the most frequently studied facial changes in recent decades, due to their high incidence. The aim of this study was to identify the application of the nursing process applied to patients with cleft lip and/or palate, described in the literature. It is an integrative review of a qualitative approach focused on the search for results for evidence-based practice. From the combination of the descriptors, 38 productions were located. After applying the established criteria, the final sample consisted of seven articles. the implementation of a care protocol for patients with cleft, in different institutional contexts, and the results found in this review may also inspire the realization of future studies that demonstrate the impact that nursing care has on the rehabilitation of these patients.

Keywords: cleft lip, cleft palate, nursing process, nursing diagnosis, nursing.

\section{INTRODUÇÃO}

As fissuras ou fendas labiopalatinas são malformações ocasionadas a partir da não junção ou da junção incompleta dos ossos faciais durante a vida embrionária e estão entre as anomalias congênitas mais comuns e se originam entre a 4 a e a 12a semana, sendo a fissura labial mais comum até a 8 a semana e a palatina até a 12 a de gestação'.

As fissuras labiopalatinas são consideradas as alterações de face mais frequentemente estudadas nas últimas décadas, em razão de sua grande incidência, especialmente no Brasil, onde a ocorrência é de 1:673 nascimentos ${ }^{2}$.

As fissuras são classificadas de acordo com as estruturas que acometem, tendo como ponto de referência o forame incisivo. As fissuras que recebem classificação de pré-forame incisivo são aquelas que acometem estruturas anteriores ao forame incisivo, ou seja, o palato primário, envolvendo lábio e/ou rebordo alveolar, podendo ser unilateral (direita ou esquerda), bilateral ou mediana - dependendo da extensão em que 
ocorre em cada uma dessas classificações, a fissura pode ser considerada completa ou incompleta. Quando entre as estruturas acometidas estão o palato primário e secundário, se estendendo do lábio até a úvula e atravessando o rebordo alveolar classifica-se como fissura transforame incisivo, também podendo ser unilateral, bilateral ou mediana. Podemos classificá-las também como pós-forame incisivo em situações do acometimento de estruturas posteriores ao forame incisivo, ou seja, o palato secundário, podendo ser completa ou incompleta dependendo da extensão anatômica atingida ${ }^{3}$.

Para melhor promover a qualidade de vida dos portadores pode-se realizar correção, que são cirurgias durante o tratamento. Essas correções objetivam o aspecto estético do nariz e do lábio, assim como normalizar a fala e a linguagem, melhorar a permeabilidade das vias áreas e devolver a função mastigatória ${ }^{4}$.

No Brasil, a lei no 1.172 de 2015 dispõe sobre a obrigatoriedade da cirurgia reparadora de lábio leporino ou fenda palatina no Sistema Único de Saúde (SUS) e nos conveniados e dá outras providências como serviços de fonoaudiologia, psicologia e ortodontia ${ }^{4}$.

No Brasil a proporção é de uma criança fissurada para cada 650 nascimentos. Estudos demonstram que fatores genéticos e ambientais podem influenciar na formação das fissuras. Estimativas feitas em várias populações revelam que cerca de $20 \%$ dos casos totais de fissuras labiopalatinas são familiais. Nestes casos em que há recorrência familiar, prevê-se que a contribuição genética seja mais predominante, mas a incidência varia de acordo com a localização geográfica, etnia e condição socioeconômica ${ }^{5,6}$.

O nascimento de uma criança com fissura pode provocar uma crise que atinge toda a família, abalando sua identidade, estrutura e funcionamento. Por vezes, esta família está despreparada para enfrentar, já que o filho com deficiência representa quebra de expectativas. A vida familiar sofre alterações frente às exigências emocionais e à convivência com a criança, gerando conflitos e levando à instabilidade emocional, alteração no relacionamento do casal e distanciamento entre seus membros. Vale considerar também o custo financeiro do tratamento, o qual nem sempre se encaixa nas condições socioeconômicas da família ${ }^{7}$.
A atuação do enfermeiro é primordial na assistência ao paciente portador da fissura labiopalatina, devendo aplicar o processo de enfermagem a todo momento, e neste contexto, este é o profissional que deve possuir o conhecimento e ser capaz de atender da melhor forma possível as necessidades dessa anomalia congênita ${ }^{8}$.

Dito isso, o objetivo desse estudo foi identificar a aplicação do processo de enfermagem aplicados a pacientes com fissura de lábio e/ou palato, descrita na literatura.

\section{METODOLOGIA}

Trata-se de revisão integrativa de abordagem qualitativa com foco na busca de resultados para a prática baseada em evidências. Compreende-se prática baseada em evidencias a abordagem que possibilita melhorias na qualidade assistencial em saúde, envolve a busca e a avaliação críticas de evidências científicas disponíveis para a tomada de decisão sobre a assistência com foco na intensificação do julgamento clínico? .

Neste trabalho a revisão integrativa é útil para integrar informações de estudos já previamente realizados evidenciando as práticas efetivas e eliminando as prejudiciais, aproximando a evidência com sua aplicação no cuidado $^{10}$.

A elaboração da pergunta de pesquisa seguiu-se pela estratégia da metodologia do acrônimo PICOS (população, intervenção ou exposição, comparação, desfecho e estudo), sendo que os itens de comparação não foram aplicados, conforme apresentado no quadro ${ }^{11}$.

Quadro 1. Estratégia PICOS para construção de pergunta norteadora.

\begin{tabular}{cll}
\hline P & População & $\begin{array}{l}\text { Pessoa com fissura de lábio } \\
\text { e/ou palato } \\
\text { Aplicação do processo de } \\
\text { enfermagem }\end{array}$ \\
I & Intervenção & - \\
C & Comparação & - \\
O & Desfecho & \multicolumn{1}{c}{ - } \\
S & Tipos de Estudo & Descritivos e experimentais \\
\hline
\end{tabular}

Desta forma a pergunta de pesquisa se constrói: Quais as evidências sobre a aplicação do processo de enfermagem a pessoa com fissura de lábio e/ou palato?

Optou-se pela metodologia da revisão sistemática de literatura para resolução do 
objetivo proposto, este método compreende um traçado de estudos científicos publicados utilizando as seguintes etapas: (1) elaboração da pergunta de pesquisa; (2) busca na literatura; (3) seleção dos artigos; (4) extração dos dados; (5) avaliação da qualidade metodológica; (6) síntese dos dados; (7) avaliação da qualidade das evidências; (8) redação e publicação dos resultados obtidos 9 .

A busca de literatura foi realizada nas seguintes bases de dados: Biblioteca Virtual de saúde (BVS), Scielo, Lilacs, PubMed e CAPES. As palavras chave apoiadas nos Descritores em Ciências da Saúde (DeCs) foram: Processo de enfermagem; fissura lábio e/ou palato; diagnósticos de enfermagem, intervenções de enfermagem, Assistência de enfermagem.

Para selecionar os artigos de interesse no estudo e que respondessem o objetivo, foram adotados os seguintes critérios de inclusão: artigos de periódicos com abordagens metodológicas de estudos descritivos; estudos de casos e observacionais, com ou sem randomização, de abordagem quantitativas ou qualitativas, publicados no idioma português, inglês e espanhol. Não foi estabelecido recorte temporal.

\section{RESULTADOS}

A partir da combinação dos descritores com utilização dos qualificadores "AND" e "OR", foram localizadas 38 produções. Após aplicação dos critérios estabelecidos, a amostra final foi constituída de sete artigos.

A análise e interpretação dos dados foi realizada organizadamente e sintetizada por meio da elaboração do quadro sinóptico que foi elaborado com os seguintes itens: identificação do estudo; autores; delineamento do estudo, participantes da pesquisa; resultados e recomendações.

Os artigos selecionados para esta revisão foram analisados na íntegra e agrupados nas áreas temáticas.

\section{DISCUSSÃO}

$O$ processo de enfermagem possibilita ao enfermeiro a elaboração de uma prescrição de Enfermagem com cuidados individualizados, além de viabilizar a melhoria nos registros de Enfermagem e a humanização da assistência ${ }^{12}$.
Porém, estudo ${ }^{19}$ revela que muitos profissionais encontram dificuldades em colocar o processo de enfermagem em prática e algumas vezes não o aplica, isso devido à mecânica dos afazeres rotineiros, à demanda de trabalho, à falta de apoio institucional, à falta de profissionais, e à sobrecarga de trabalho.

Pode-se dizer que o processo de enfermagem é um instrumento que tem a função de organizar a assistência de Enfermagem.

A execução do processo de enfermagem como ações de cuidado rotineiro pelos enfermeiros é representada por ser um cuidado direcionado por ações de natureza prescritiva que, na maioria das vezes, têm resolução imediata, priorizadas em relação às condições pendentes, às necessidades e condições de risco, havendo pouco tempo para a escuta atenta ou relação de proximidade com os sujeitos do cuidado $^{13}$.

O Processo de enfermagem deve ser aplicado desde o momento pré-operatório do recém-nascido portador de fissura. 0 planejamento, assim como a implementação das intervenções devem ser focadas na nutrição da criança, assim como no apoio psicológico a mãe ${ }^{14}$. Quando se reúne literatura acerca do assunto a predominância de cuidados é quanto à alimentação.

Os desafios na alimentação do neonato dependem da complexidade da fissura, podendo esta resultar em sucção inadequada por falta de pressão intraoral, tempo prolongado e regurgitação. Nesse contexto é mencionado ainda que o aleitamento materno deve ser incentivado e dentre suas intervenções o enfermeiro deve realizar orientações ${ }^{20}$.

Além da alimentação, a aplicação do processo de enfermagem ao paciente com fissura é ampla, e esse processo deve acontecer a todo momento com o paciente internado, assim como a sua família, devendo a assistência aborda-lo em internação, unidade semi-intensiva, unidade intensiva, ambulatório, e até mesmo na Central de Material e Esterilização ${ }^{15}$. 
Quadro 2. Artigos inclusos na revisão integrativa.

\begin{tabular}{|c|c|c|c|}
\hline Artigos & Tipo de estudo & Participantes & Resultados \\
\hline $\begin{array}{l}\text { Souza et } \\
\text { al. }^{12}\end{array}$ & Qualitativo & $\begin{array}{l}11 \text { enfermeiros e } 26 \\
\text { técnicos e auxiliares } \\
\text { de Enfermagem }\end{array}$ & $\begin{array}{l}\text { O processo de enfermagem é concebido como um } \\
\text { instrumento para organizar a assistência e prescrever } \\
\text { os cuidados de Enfermagem. As rotinas de trabalho } \\
\text { evidenciam as atividades técnicas. As expectativas com } \\
\text { a implementação do processo de enfermagem } \\
\text { envolvem a melhoria da qualidade da assistência }\end{array}$ \\
\hline $\begin{array}{l}\text { Benedet } \\
\text { et al. }^{13}\end{array}$ & $\begin{array}{l}\text { Qualitativo com } \\
\text { caráter descritivo } \\
\text { e exploratório }\end{array}$ & 38 enfermeiros & $\begin{array}{l}\text { O Processo de Enfermagem melhora a sistematização } \\
\text { da assistência de enfermagem, com a filosofia da } \\
\text { Instituição e do Serviço de Enfermagem alinhadas a } \\
\text { uma política de educação permanente. }\end{array}$ \\
\hline $\begin{array}{l}\text { Bueno et } \\
\text { al. }^{14}\end{array}$ & Descritivo & $\begin{array}{l}\text { Pacientes do } \\
\text { Hospital de Pesquisa } \\
\text { e Reabilitação de } \\
\text { Lesões Lábio } \\
\text { Palatais da USP } \\
\text { Bauru }\end{array}$ & \\
\hline Fontes ${ }^{15}$ & $\begin{array}{l}\text { Descritivo, } \\
\text { exploratório }\end{array}$ & $\begin{array}{l}40 \text { pacientes no pré } \\
\text { e pós operatório de } \\
\text { cirurgia plástica } \\
\text { eletiva }\end{array}$ & $\begin{array}{l}\mathrm{Na} \text { fase pré-operatória foram identificadas } 29 \\
\text { categorias diagnósticas e, na fase pós, } 26 \text {. A média de } \\
\text { diagnósticos por paciente na fase pré foi de } 4,7( \pm 1,9) \\
\text { e na fase pós } 6,7\end{array}$ \\
\hline $\begin{array}{l}\text { Mondini } \\
\text { et al. }^{16}\end{array}$ & $\begin{array}{l}\text { Descritivo, } \\
\text { exploratório }\end{array}$ & $\begin{array}{l}\text { Pacientes em } \\
\text { tratamento no } \\
\text { HRAC USP }\end{array}$ & $\begin{array}{l}\text { O planejamento dos cuidados de enfermagem é } \\
\text { baseado nos referenciais da NANDA Internacional e as } \\
\text { intervenções na NIC }\end{array}$ \\
\hline $\begin{array}{l}\text { Ranção et } \\
\text { al. }^{17}\end{array}$ & $\begin{array}{c}\text { Descritivo, } \\
\text { observacional }\end{array}$ & $\begin{array}{l}1 \text { Criança em pré- } \\
\text { operatório para } \\
\text { realização de } \\
\text { cirurgia plástica } \\
\text { para correção de } \\
\text { fenda palatina }\end{array}$ & $\begin{array}{l}\text { A aplicação do processo de enfermagem contribuiu } \\
\text { para uma assistência de qualidade e para a alta } \\
\text { hospitalar. }\end{array}$ \\
\hline $\begin{array}{l}\text { Medeiros } \\
\text { et al. }^{18}\end{array}$ & $\begin{array}{l}\text { Revisão de } \\
\text { literatura }\end{array}$ & & $\begin{array}{l}62,5 \% \text { dos artigos selecionados foram da região } \\
\text { sudeste. Esta região possui maior índice da patologia } \\
\text { observada nos registros. Dos artigos selecionados } \\
87,5 \% \text { estimulam a manutenção do aleitamento } \\
\text { materno na alimentação dos lactentes e neonatos } \\
\text { portadoras desta doença. Abordaram também a } \\
\text { atuação do enfermeiro diante o diagnóstico da } \\
\text { patologia e das orientações a serem feitas aos pais. }\end{array}$ \\
\hline
\end{tabular}

O processo de enfermagem dever ser sustentado nas fases da Sistematização da Assistência de Enfermagem (SAE), fundamentada na Teoria das Necessidades Humanas Básicas de Horta e na teoria do autocuidado de Orem. O uso de uma linguagem padronizada como a classificação internacional de diagnósticos, intervenções e resultados de enfermagem (NANDA, NIC e NOC) é um importante instrumento para a assistência, pesquisa e ensino na enfermagem. $O$ seu uso possibilita a organização e o resgate dos dados documentados. Os diagnósticos, enquanto processo de julgamento clínico, são conteúdos essenciais que devem figurar nos sistemas de informação em saúde ${ }^{15}$.

$O$ processo de enfermagem quando bem aplicado em todas suas etapas propicia um olhar sobre o cuidado singular, levando-se em conta as peculiaridades envolvidas no tratamento, além de contribuir para a melhor compreensão do papel do enfermeiro no cuidado do paciente e nas orientações a família ${ }^{18}$.

Após análise dos estudos ${ }^{14-18,}$ os autores estabeleceram os seguintes dados. 
Para construir o histórico do paciente, a anamnese deve ser profunda e extensa, não só com o paciente, mas também com seu meio familiar.

É fundamental que o enfermeiro assista e avalie o seu cliente numa visão holística no tocante aos aspectos biopsicossociais e espirituais. Quando o corpo e ou a mente sofrem, a pessoa é afetada em sua totalidade. Não se deve, portanto, enfocar apenas as partes que incomodam o indivíduo ${ }^{21}$.

É extremamente importante que o enfermeiro tenha conhecimento técnico e científico para assistir o seu cliente, resgatando os princípios de necessidades humanas básicas citados em algumas teorias de enfermagem. Ao realizar uma boa anamnese torna-se possível aumentar o elo de confiança entre profissional de saúde e cliente, coletar dados indispensáveis para o seu tratamento, identificar sinais e sintomas ${ }^{21}$.

Após histórico, tem-se reunidas informações necessárias para o estabelecimento de diagnósticos de enfermagem, conforme a taxonomia NANDA. Abaixo estão as principais categorias diagnósticas e intervenções encontradas nessa revisão. Vale ressaltar que os autores usaram da Classificação de Intervenções de Enfermagem (NIC) para complementação.

Quadro 3. Diagnósticos e intervenções de enfermagem descritos na literatura.

\begin{tabular}{|c|c|}
\hline $\begin{array}{l}\text { Diagnósticos de } \\
\text { enfermagem }\end{array}$ & Intervenções de enfermagem \\
\hline Risco de Infecção & $\begin{array}{l}\text { - Fazer a higiene oronasal com cotonete molhado em água fervida, antes e } \\
\text { depois da alimentação, para que se evite a permanência de resíduos e } \\
\text { partículas de leite na região }\end{array}$ \\
\hline $\begin{array}{l}\text { Baixa autoestima } \\
\text { situacional }\end{array}$ & $\begin{array}{l}\text { - } \text { Determinar a confiança do paciente no próprio julgamento } \\
\text { - } \quad \text { Encorajar o paciente a identificar seus pontos positivos } \\
\text { - } \\
\text { - } \\
\text { Feforçar os pontos positivos identificados pelo paciente } \\
\text { - } \text { apropriado } \\
\text { - } \\
\text { - } \\
\text { Refrear as críticas ne pefrear provociencias que aumentem a autonomia do paciente, conforme }\end{array}$ \\
\hline Risco de aspiração & $\begin{array}{l}\text { - } \quad \text { Manter a criança em posição semi-fowler ao oferecer alimentos; } \\
\text { - } \quad \text { Realizar pausas durante a mamada para favorecer a eructação; } \\
\text { - } \quad \text { Para exercitar a musculatura oral, proporcionar estímulos no lado da fissura } \\
\text { - } \quad \text { através do contato com o bico do seio ou da mamadeira } \\
\text { - Colocar a criança em decúbito lateral, após a mamada }\end{array}$ \\
\hline $\begin{array}{l}\text { Membrana mucosa } \\
\text { oral prejudicada }\end{array}$ & $\begin{array}{l}\text { - Oferecer dieta fria, liquida ou pastosa; } \\
\text { - } \quad \text { Realizar higiene oral }\end{array}$ \\
\hline Dor & $\begin{array}{l}\text { - } \quad \text { Avaliar a dor quanto à localização, frequência e duração; } \\
\text { - } \quad \text { Avaliar a eficácia das medidas de controle da dor; } \\
\text { - } \quad \text { Favorecer repouso/sono adequados para o alívio da dor; } \\
\text { - } \quad \text { Investigar a experiência de dor da criança; } \\
\text { - } \quad \text { Preparar a criança para procedimento de administração de medicamento; } \\
\text { - } \quad \text { Ensinar o uso de técnicas não farmacológicas (relaxamento, imagem orientada, } \\
\text { musicoterapia, diversão, aplicação de compressas frias/quentes, aplicação de } \\
\text { - } \quad \text { massagem) antes, após e se possível durante o momento de dor; } \\
\text { duração, quando necessário. }\end{array}$ \\
\hline $\begin{array}{l}\text { Padrão de Sono } \\
\text { Prejudicado }\end{array}$ & $\begin{array}{l}\text { - } \quad \text { Monitorar o padrão de sono; } \\
\text { - } \quad \text { Registrar o padrão do sono e quantidade de horas dormidas; } \\
\text { - } \quad \text { Identificar e reduzir estressores ambientais; } \\
\text { - } \quad \text { Planejar as rotinas de cuidados de enfermagem para que procedimentos }\end{array}$ \\
\hline
\end{tabular}


desagradáveis ou dolorosos não ocorram após as $20 \mathrm{~h}$;

- Observar as circunstâncias físicas - apneia do sono, nesse caso por via aérea obstruída;

- Proporcionar um ambiente calmo e seguro;

- Orientar a acompanhante a manter regularidade nos horários de deitar

Integridade Tissular - Massagear área em torno da fissura para estimular circulação;

Prejudicada

- Manter técnica asséptica no curativo ao fazer os cuidados da fissura;

- examinar/observar monitorar as condições locais da pele como ruptura, cor, calor, textura, edemas, ulcerações, pontos de pressão, infecção, áreas de necrose, fontes de pressão e fricção;

\begin{tabular}{|c|c|}
\hline $\begin{array}{l}\text { Integridade da pele } \\
\text { prejudicada }\end{array}$ & $\begin{array}{l}\text { - Estimular a mudança de posição; } \\
\text { - Explicar cuidados com a fissura, pele e áreas circunvizinhas; } \\
\text { - Manter a pele limpa e seca; } \\
\text { - } \quad \text { Orientar higiene corporal e oral; } \\
\text { - Supervisionar o cuidado com a pele; } \\
\text { - } \quad \text { Edidar do sítio de inserção de dispositivos invasivos; } \\
\text { - } \quad \text { Controlar a ingestão e a excreta - Balanço hídrico; } \\
\text { - Estimular a hidratação; } \\
\text { - Realizar massagem na pele. }\end{array}$ \\
\hline $\begin{array}{c}\text { Risco de } \\
\text { recuperação } \\
\text { cirúrgica retardada }\end{array}$ & $\begin{array}{l}\text { - Observar e registrar sinais de infecção; } \\
\text { - Avaliar a ferida cirúrgica incluindo localização e tamanho; } \\
\text { - Orientar o paciente e familiar o motivo do aumento de dias de internação } \\
\text { hospitalar }\end{array}$ \\
\hline $\begin{array}{c}\text { Déficit de } \\
\text { conhecimento }\end{array}$ & $\begin{array}{l}\text { - Estabelecer um relacionamento de confiança com o paciente e familia para } \\
\text { facilitar a aprendizagem; } \\
\text { - Orientar paciente e/ou acompanhante sobre a fissura; Ensinar o paciente e } \\
\text { familiar as habilidades necessárias ao seu estilo de vida diário; }\end{array}$ \\
\hline
\end{tabular}

Nota-se que há um grande déficit de estudos que contemplem diagnósticos e intervenções de enfermagem aplicados ao paciente portador de fissura labiopalatina. Todavia, os estudos existentes são capazes de preencher parte da lacuna existente podendo trazer contributos para a implementação de um protocolo de cuidados ao portador de fissura, em diferentes contextos institucionais, e os resultados encontrados nessa revisão podem também inspirar a realização de futuros estudos que demonstrem o impacto que a assistência de enfermagem tem na reabilitação desses pacientes.

É perceptível, a partir da análise dos estudos, que quando o enfermeiro é capaz de aplicar os diagnósticos e intervenções adequadas ele proporciona ao portador maior bem-estar, diminuição do tempo de internação e maior qualidade de vida.

\section{CONFLITO DE INTERESSE}

Os autores declaram não haver qualquer potencial conflito de interesse que possa interferir na imparcialidade deste trabalho científico.

\section{REFERÊNCIAS}

1. Costa RR, Takeshita WM, Farah GJ. Levantamento epidemiológico de fissuras labiopalatais no município de Maringá e região. Rev Assoc Paul Cir Dente. 2013; 67(1):40-4.

2. Di Ninno CQMS, Fonseca LFN, Pimenta MVE, Vieira ZG, Fonseca JA, Miranda ICC et al. Levantamento epidemiológico dos pacientes portadores de fissura de lábio e/ou palato de um centro especializado de Belo Horizonte. Rev CEFAC. 2011;13(6):1002-8 DOI: https://doi.org/10.1590/S1516-18462011005000046.

3. Faraj JO, Romanelli A, André M. Alterações dimensionais transversas do arco dentário com fissura labiopalatina, no estágio de dentadura decídua. Rev Dent Press Ortodon Ortop Facial. 2007;12(5):100-8 DOI: $\quad$ https://doi.org/10.1590/S141554192007000500013. 
4. Miachon MD, Leme PL. Tratamento operatório das fendas labiais. Rev Col Bras Cir 2014;41(3):208-14 DOI: https://doi.org/10.1590/S0100-69912014000300013.

5. Brito L, Bueno DF, Bertola D, Burin B, Alonso N, Passos-Bueno MR. A contribuição de fatores genéticos e ambientais para a ocorrência das fissuras lábiopalatinas não-sindrômicas é a mesma em diferentes regiões do país? Rev Bras Cir Craniomaxilofac. 2008;11(3):85-8

6. Borges AR, Mariano L, Sá J, Medrado AP, Veiga PC, Reis SRA. Fissuras labiais e/ou palatinas não sindrômicas: determinantes ambientais e genéticos. Rev Bahiana Odonto. 2014;5(1):48-58 DOI: https://doi.org/10.17267/2238-

2720revbahianaodonto.v5i1.329

7. Silva CM, Locks A, Carcereri DL, Silva DGV. A escola na promoção da saúde de crianças com fissura labiopalatal. Texto Contexto-Enferm. 2013;22(4):10418. DOI: https://doi.org/10.1590/S0104$\underline{07072013000400021}$

8. Medeiros LK, Oliveira MAA, Martins TA, Gonçalves V, Cardoso IM, Santos NAR. Atuação do enfermeiro nos cuidados alimentares de crianças com fissuras lábio e/ou palatina: uma revisão da literatura. Rev Educ Meio Amb Saúde. 2018;8(1):1-12.

9. Galvão TF, Pereira MG. Revisões sistemáticas da literatura: passos para sua elaboração. Epidemiol Serv Saúde. 2014;23(1):183-4.

DOI:

http://dx.doi.org/10.5123/S1679-

\section{$\underline{49742014000100018}$}

10. Sampaio RF, Mancini MC. Estudos de revisão sistemática: um guia para síntese criteriosa da evidência científica. Rev Bras Fisioter. 2007;11(1):839. DOI: https://doi.org/10.1590/S1413$\underline{35552007000100013}$

11. Santos CMC, Pimenta CAM, Nobre MRC. A estratégia PICO para a construção da pergunta de pesquisa e busca de evidências. Rev Latino-Am Enfermagem. 2007;15(3):508-11. DOI: https://doi.org/10.1590/S0104-11692007000300023

12. Souza MFG, Santos ADB, Monteiro Al. O processo de enfermagem na concepção de profissionais de Enfermagem de um hospital de ensino. Rev Bras Enferm. 2013;66(2):167-73. DOI: https://doi.org/10.1590/S0034-71672013000200003

13. Benedet S, Gelbcke F, Amante L, Padilha M, Pires D. Processo de enfermagem: instrumento da sistematização da assistência de enfermagem na percepção dos enfermeiros. Rev Pesq Cuidado
Fundam.

2016;8(3):4780-8.

DOI:

http://dx.doi.org/10.9789/2175-5361.rpcfo.v8.4237

14. Bueno AG, Bachega MI, Thomé S. Reabilitação de fissuras lábio-palatais - Uma experiência de enfermagem. Rev Bras Enferm. 1980;33(2):242-52 DOI: https://doi.org/10.1590/0034716719800002000013

15. Fontes, Cassiana Mendes Bertoncello. Fissuras Lábio-Palatais: Diagnósticos de enfermagem no pré e pós-operatório. [Dissertação]. São Paulo: Universidade de São Paulo, Escola de Enfermagem; 2001.

16. Mondini CCSD, Trettene AS, Lisboa IA, Beraldo CC, Bertolini LRL, Shinomia MT, Herrera MCA. Assistência de enfermagem na reabilitação de pacientes com anomalias craniofaciais. Anais. 2013

17. Ranção CSF, Thiengo PCS, Peres EM, Gomes HS. Aplicação da assistência de enfermagem no pósoperatório de palatoplastia. Academus Rev Cient Saúde. 2017;2(1):1-7.

18. Medeiros L, Oliveira MAA, Martins TA, Vitor Gonçalves, Cardoso IM, Santos NAR. Atuação do Enfermeiro nos Cuidados Alimentares de Crianças com Fissuras Lábio e/ou Palatina: Uma Revisão da Literatura. REMAS - Rev Educ Meio Amb Saúde. 2018;8(1):1-12

19. Trindade L, Ferreira A, Silveira A, Rocha E. Processo de enfermagem: desafios para sua implementação sob a ótica de enfermeiros. Saúde (Santa Maria). 2016;42(1):75-82.

DOI: https://doi.org/10.5902/2236583419805

20. Santos KCR, Bohn MLS, Motta GCP, Silva EF, Lorenzini E. Cuidados à criança com fissura labiopalatina: uma revisão integrativa. Rev Pesq Cuidado Fundam. 2014;6(1):425-32. DOI: http://dx.doi.org/10.9789/2175-5361.rpcfo.v6.2953

21. Santos $N$, Veiga $P$, Andrade R. Importância da anamnese e do exame físico para o cuidado do enfermeiro. Rev Bras Enferm. 2011;64(2):355-8. DOI: https://doi.org/10.1590/S0034-71672011000200021 